\title{
Survivre au cancer colorectal
}

$\mathrm{E}$ n 2007, on diagnostiquera environ 20460 nouveaux cas de cancer colorectal au Canada et 8700 personnes mourront de cette maladie ${ }^{1}$. Presque la moitié des victimes survivraient si le Canada avait adopté une démarche plus robuste et cohérente de détection précoce et de traitement. Contrairement au cancer du poumon, autre grande cause de mortalité, le cancer du côlon est facile à détecter dès le début, le plus souvent au stade prémalin. Et contrairement à la détection précoce du cancer du sein et du cancer de la prostate, la détection précoce du cancer colorectal, particulièrement au stade de l'adénome précancéreux, entraîne régulièrement l'excision et la guérison. De plus, si le cancer colorectal est détecté précocement, le taux de mortalité est faible et $90 \%$ des patients survivent cinq ans.

On détecte la plupart des lésions chez des patients asymptomatiques au moyen d'interventions comme des tests de détection de la présence de sang occulte dans les matières fécales, une sigmoïdoscopie ou une coloscopie ${ }^{2}$. Une seule sigmoïdoscopie réduit d'environ $60 \%$ le risque de cancer colorectal distal mortel en dix ans, et de $44 \%$ le risque de cancer du côlon en six ans ${ }^{3}$. En outre, le ratio coût-efficacité différentiel de chacune des méthodes actuellement reconnues de dépistage du cancer colorectal s'établit à moins de 50000 \$ par année de vie sauvée - ce qui est inférieur à ce ratio dans le cas du cancer du col, du sein et de la prostate ${ }^{4}$.

Avec la mise en service prévue de nouvelles techniques de diagnostic comme la colographie par tomodensitométrie, l'analyse de l'ADN dans les selles et l'examen du côlon par vidéocapsule sans fil, les patients pourront à l'avenir subir des tests de dépistage avec perturbation minimale et qui pourront peut-être même être faits à domicile ou au travail. Dans l'avenir prévisible, toutefois, ces méthodes ne supplanteront pas la coloscopie conventionnelle pour distinguer les résultats vrais et faux positifs de pathologie du côlon obtenus par d'autres méthodes de dépistage. Ces nouvelles méthodes ne permettront pas non plus de surmonter les obstacles au dépistage.

Le pourcentage des patients qui subissent un test de dépistage du cancer colorectal est extraordinairement faible au Canada ${ }^{5}$. Dans ce numéro, nous présentons les résultats d'un sondage réalisé en 2003 au cours duquel 23,5\% seulement des personnes à risque moyen ont déclaré s'être soumises à un test de dépistage ${ }^{6}$. En guise de comparaison, aux États-Unis, où l'on a déployé un effort concerté pour lancer le dépistage national, 62,9\% des personnes à risque moyen se sont soumises à un test de dépistage en $2007^{7}$. Il y a aussi des programmes nationaux de dépistage en cours au Royaume-Uni et en Europe, mais le Canada tire toujours de l'arrière et compte quelques initiatives provinciales seulement (voir page 593).

Un effort concerté pour lutter contre ce cancer doit commencer par l'adoption du dépistage du cancer colorectal comme priorité nationale de la santé. Le programme cana- dien doit prévoir des ressources suffisantes et des mesures d'information des patients et des médecins. En informant les Canadiens des risques que pose le cancer colorectal, on les incitera probablement à demander un test de dépistage. Les médecins ont toutefois un rôle clé à jouer. Actuellement, la raison la plus courante pour laquelle on ne subit pas un test de dépistage, c'est que le médecin traitant ne l'a pas recommandé. (Les praticiens devraient noter que plus de la moitié de toutes les poursuites pour faute professionnelle intentées contre des médecins de première ligne dans le cas des maladies gastro-intestinales découlent d'un cancer colorectal diagnostiqué tardivement.)

Jusqu'à ce qu'un programme national actif soit en place, il revient à chaque médecin d'agir. Plusieurs sociétés professionnelles et fondations sont disposées à prêter leur concours, mais les médecins doivent d'abord s'attaquer à leur propre ignorance et surmonter leur réticence, et accepter leur obligation de commencer à informer leurs patients et de les persuader de subir le test de dépistage.

Nous pouvons freiner cette maladie mortelle et nous en avons les moyens. Il ne nous manque que de l'information et la volonté nationale d'agir. Il est abondamment clair que si l'on maintient le cap actuel, c'est-à-dire celui de l'inaction, cette maladie continuera de coûter des milliers de vie.

\section{Alan Barkun MDCM MSc}

Titulaire de la Chaire Douglas-G.-Kinnear de gastroentérologie

Directeur de la Division de gastro-entérologie

Université McGill et Centre universitaire de santé McGill Montréal, Que.

\section{Ken Flegel MDCM MSc}

Rédacteur associé principal, JAMC

Avec l'équipe de rédaction de l'éditorial (Paul C. Hébert MD MHSc, Matthew Stanbrook MD PhD, Barbara Sibbald BJ, Noni MacDonald MD MSc et Amir Attaran LLB PhD)

\section{RÉFÉRENCES}

I. Statistiques canadiennes sur le cancer 2007. Toronto : Société canadienne du cancer/ Institut national du cancer du Canada; 2007. p. I5. Disponible : http://129.33.170.32 /ccs/internet/standard/o,3182,3172_I4279__langId-fr,oo.html (consulté le 2 août 2007).

2. Ferraris R, Senore C, Fracchia M, et al. Predictive value of rectal bleeding for distal colonic neoplastic lesions in a screened population. Eur J Cancer 2004;40:245-52.

3. Muller AD, Sonnenberg A. Prevention of colorectal cancer by flexible endoscopy and polypectomy: a case-control study of 32702 veterans. Ann Intern Med I995;I23:904-Io.

4. Barkun AN, Jobin G, Cousineau G, et al. The Quebec Association of Gastroenterology position paper on colorectal cancer screening-2003. Can J Gastroenterol 2004; I8:509-I9.

5. Rabeneck L, Paszat LF. A population-based estimate of the extent of colorectal cancer screening in Ontario. Am J Gastroenterol 2004;99:II4I-4.

6. Zarychanski R, Chen $\mathrm{Y}$, Bernstein $\mathrm{CN}$, et al. Frequency of colorectal cancer screening and the impact of family physicians on screening behaviour. JAMC 2007;177:593-7.

7. Peterson NB, Murff HJ, Ness RM, et al. Colorectal cancer screening among men and women in the United States. J Womens Health (Larchmt) 2007;16:57-65 